06

\title{
Магнитные превращения и полиморфный переход ферромагнитных сталей при ударно-волновом нагружении
}

\author{
() А.М. Молодец, А.А. Голышев, А.Н. Емельянов, А.А. Козлов \\ Институт проблем химической фризики РАН, \\ 142432 Черноголовка, Россия \\ e-mail: molodets@icp.ac.ru
}

Поступило в Редакцию 9 сентября 2020 г.

В окончательной редакции 17 ноября 2020 г.

Принято к публикации 28 ноября 2020 г.

\begin{abstract}
Представлена методика регистрации магнитного превращения ферромагнитных сталей при ударноволновом нагружении. Рассмотрено функционирование датчика магнитного превращения в области давлений полиморфного перехода железа АРМКО и корпусной стали атомных реакторов 15Х2НМФА при ударноволновом нагружении. Показано, что давления магнитных превращений сталей коррелируют с давлениями полиморфных переходов этих сталей. Проведено сравнение показаний датчика и параметров полиморфных переходов железа и реакторной стали при ударно-волновом сжатии и последующей разгрузке.
\end{abstract}

Ключевые слова: железо, полиморфизм, корпусная сталь, ударные волны, магнитные превращения, ферромагнетизм.

DOI: $10.21883 /$ JTF.2021.05.50692.263-20

\section{Введение}

Как хорошо известно, в области давлений $\sim 12 \mathrm{GPa}$ железо и многие стали испытывают полиморфный переход из фазы с объемно-центрированной кубической решеткой (ОЦК) в фазу с гексагональной плотноупакованной (ГПУ) решеткой. Полиморфный ОЦК $\rightarrow$ ГПУ переход в железе происходит как при статических, так и при динамических давлениях.

Факт полиморфного ОЦК $\rightarrow$ ППУ перехода и его параметры в ударно-сжатых сталях могут быть определены и обычно определяются с помощью таких методик как методика измерения кинематических характеристик ударной волны [1], метод манганинового датчика динамического давления [2], лазерная интерферометрия скорости свободной поверхности VISAR (см. [3], ссылки в ней и ее цитирование), а в последнее время метод рентгеноструктурного анализа непосредственно во время ударного сжатия (см. [4], ссылки в ней и ее цитирование).

Следует отметить, что перечисленные методики, как правило, трудоемки и зачастую требуют дорогостоящего оборудования. В такой ситуации разработка методов экспресс-анализа ОЦК ГПУ превращения различных сталей в условиях сильного ударно-волнового сжатия представляется оправданной. В связи с этим примем во внимание особенность перехода ОЦК $\rightarrow$ ПУ в сталях, которая заключается в том, что ОЦК фаза является магнитной, а ГПУ фаза оказывается немагнитной. Поэтому в условиях микросекундных ударно-волновых воздействий размагничивание стального образца обусловливает возникновение сильных электромагнитных импульсов [5]. Эти импульсы обычно рассматриваются как помехи $([2,6])$ и, например, в [2] был предложен способ их подавления.

Вместе с тем очевидно, что электромагнитный сигнал,

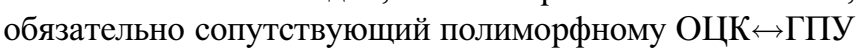
переходу в железе и во многих магнитных сталях, может быть использован в методическом отношении для регистрации изменения магнитных свойств стали и косвенно самого перехода. Однако в доступной литературе описания подобной методики не обнаружено. В настоящей работе представлена методика регистрации магнитного превращения ударно-сжимаемых ферромагнитных сталей и иллюстрация ее возможностей на примере железа АРМКО как тестового материала и корпусной стали атомных реакторов 15Х2НМФА при их ударно-волновом нагружении.

\section{1. Конструкция и ударно-волновое нагружение датчика магнитного превращения}

Полезный сигнал датчика магнитного превращения (далее просто датчика) возникает вследствие изменения магнитного потока через контур, содержащий чувствительный элемент исследуемого образца, материал которого изменяет свои магнитные свойства при ударноволновом нагружении.

Чувствительный элемент используемого датчика 1 (рис. 1,a) представляет собой зигзагообразную ленту исследуемого материала. К концам датчика точечной сваркой присоединены фольговые медные тоководы 2. В целом датчик 1 и тоководы 2 расположены в плоскости, параллельной фронту нагружающей ударной волны. В этой же плоскости рядом с датчиком 1 располагается 
манганиновый датчик давления 3 со своими медными тоководами.

Схема плоского одномерного ударно-волнового нагружения датчика представлена на рис. $1, b$. Здесь ударник 1 генерирует в металлической пластине 2 одномерный ударно-волновой импульс, который в дальнейшем приводит к реверберации волны сжатия в „мягком“ слое 3, зажатом между двумя ,жесткими“ пластинами 2 и 4. Эта реверберация аналогично [7] обусловливает ступенчатое увеличение давления в слое 3 , т.е. его ступенчатое ударное сжатие. В свою очередь реверберация плоских волн напряжения возникает на каждой ступени и в чувствительных элементах датчика 5 и манганинового датчика 6 , расположенных в этом слое. В фазе разгрузки происходит плавное уменьшение давления в 5 и 6 . Таким

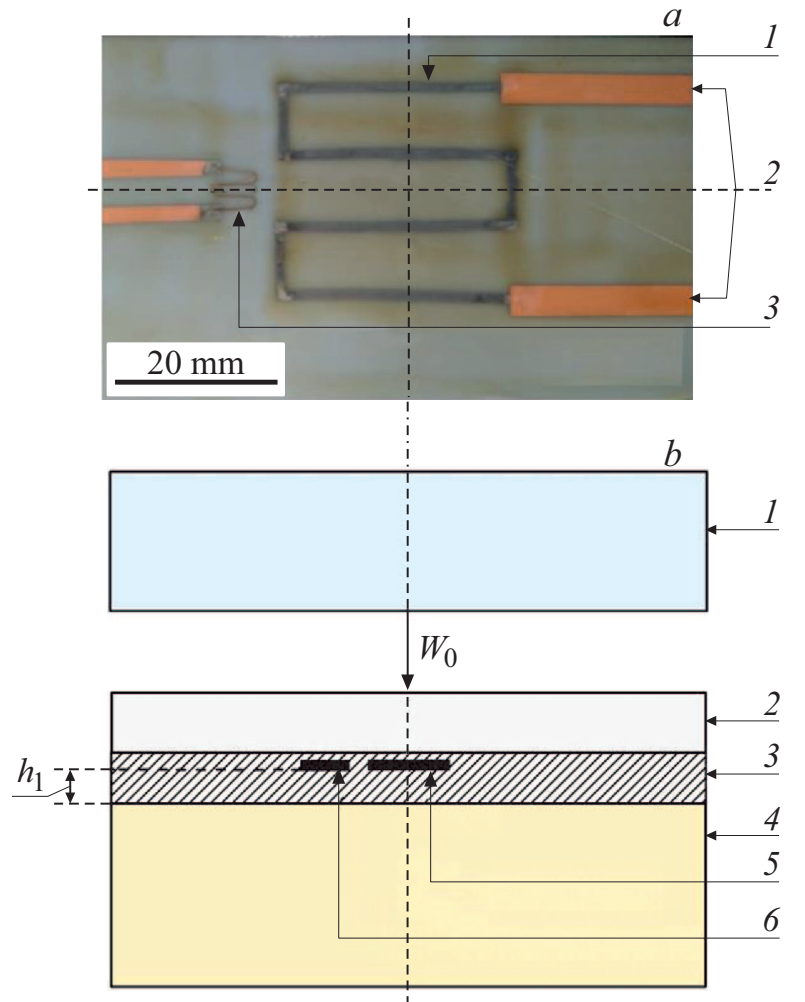

Pис. 1. Конструкция датчика и ударно-волновое нагружение измерительной ячейки. $a-$ измерительная ячейка датчика магнитного превращения: 1 - чувствительный элемент используемого датчика толщиной $0.06 \mathrm{~mm}, 2-$ медные тоководы толщиной $0.03 \mathrm{~mm}, 3$ - манганиновый датчик давления толщиной $0.06 \mathrm{~mm} ; b-$ плоское одномерное ударно-волновое нагружение измерительной ячейки, 1 - дискообразный алюминиевый ударник толщиной $7.0 \mathrm{~mm}$ с плоской частью диаметром $60 \mathrm{~mm}$, разогнанный продуктами взрыва до скорости $W_{0}=2.3(1) \mathrm{km} / \mathrm{s}$, 2 - вольфрамовая пластина толщиной $1.45(1) \mathrm{mm}, 3$ - изолирующий материал (тефлоновые пленки, склеенные вакуумной смазкой) толщиной $h_{0}=2.45(1) \mathrm{mm}, 4-$ стальная (сталь $12 \mathrm{X} 18 \mathrm{H} 10 \mathrm{~T})$ пластина из немагнитного материала толщиной $4 \mathrm{~mm}, 5$ - чувствительный элемент датчика магнитного превращения, 6 - чувствительный элемент манганинового датчика давления. 5 и 6 расположены в одной плоскости на расстоянии $h_{1}=1.75(1) \mathrm{mm}$ от поверхности 4 .
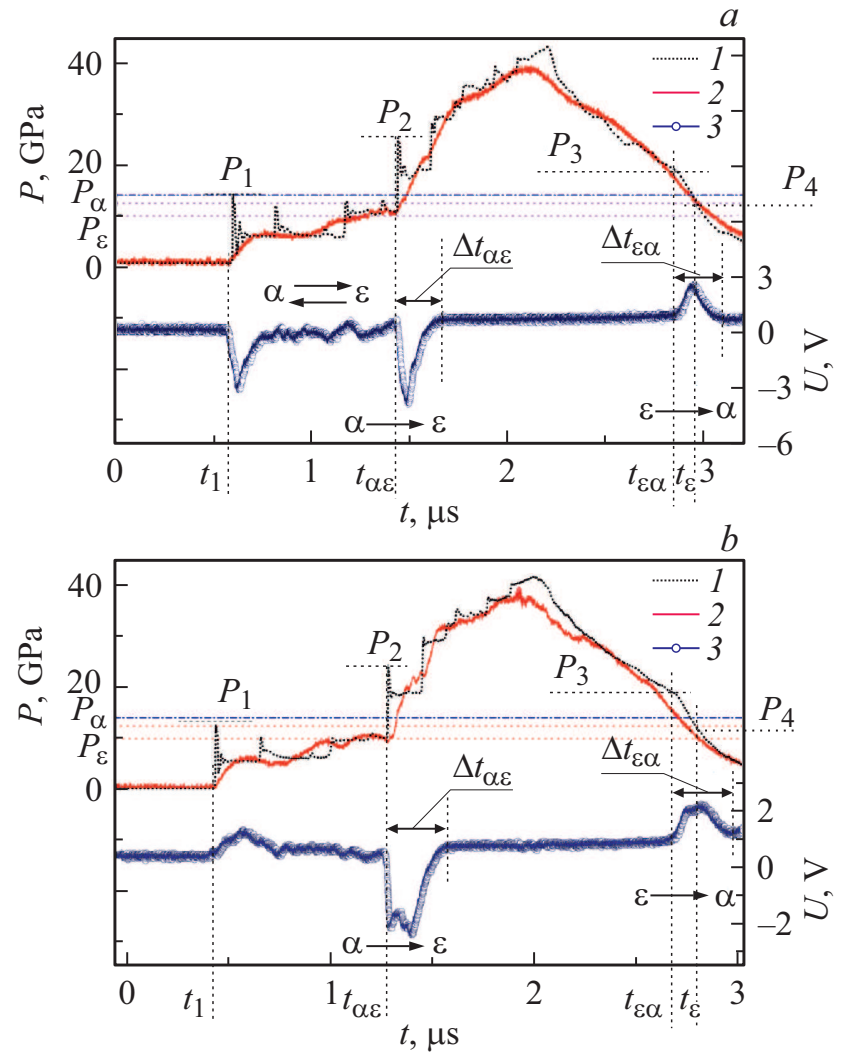

Рис. 2. Ударно-волновое нагружение и функционирование датчика магнитного превращения, содержащего чувствительный элемент из железа АРМКО $(a)$ и стали 15Х2НМФА $(b)$. 1 - расчетный профиль давления, 2 - профиль давления в изолирующем материале, 3 - показания $U$ датчика магнитного превращения. Смысл обозначений на $a, b$ идентичен. Штрихпунктирная горизонтальная линия $P_{\alpha}=13.9 \mathrm{GPa}$ - давления начала прямого ОЦК $\rightarrow$ ГПУ превращения железа при ударноволновом нагружении из [2], верхняя и нижняя пунктирные линии $P_{\varepsilon}$ - давление начала обратного ГПУ $\rightarrow$ ОЦК превращения железа при ударно-волновом нагружении соответственно из [2] $\left(P_{\varepsilon}=12.3 \mathrm{GPa}\right)$ и [11] $\left(P_{\varepsilon}=9.8 \mathrm{GPa}\right)$. Остальные обозначения - пояснения в тексте.

образом, профиль (изменение во времени) давления $P(t)$ в исследуемом материале имеет вид ступеней, на каждой из которых происходят высокочастотные колебания давления с последующей плавной фазой уменьшения давления.

На рис. 2 профиль 1 представляет количественную иллюстрацию описанной выше качественной картины. Расчет проведен в рамках разработанного ранее гидрокода (программного обеспечения для численного моделирования потоковых процессов) аналогично [8] с уравнением состояния железа из [9] и упруго-пластической моделью материала [10]. На рис. 2 графиком 1 показан расчетный профиль $P(t)$ в датчике, нагружаемом в эксперименте по схеме рис. 1.

Такой же расчет был проведен и для манганинового датчика, для которого были получены аналогичные 
модельные профили давления. Однако на экспериментальном профиле давления 2, полученном с помощью манганинового датчика 6 , отсутствуют высокочастотные колебания на ступенях давления. Это несоответствие обусловлено ограниченными частотными характеристиками манганиновой методики. Поэтому экспериментальный график 2 в настоящей работе используется главным образом в качестве отметчика времени прихода ударно-волновых возмущений в плоскость расположения датчиков.

\section{2. Электрическая оснастка датчика}

Электрическая цепь датчика идентична электрической цепи измерения электросопротивления манганинового датчика при ударно-волновом нагружении (см., например, описание в [12]) с использованием мостовой схемы Уитстона. Номиналы мостовой схемы, используемой в настоящей работе, приведены на рис. 3. Здесь чувствительный элемент датчика $R_{S} \approx 0.6 \Omega$ включен в левое плечо моста $R_{S}-R_{1}-R_{2}-R_{3}$ через высокочастотный кабель РК-75. Номинал сопротивления $R_{1}$ составляет $R_{1}=1.8 \Omega$. Номинал балластного сопротивления $R_{d}$, включенного последовательно $R_{S}$, выбирается как $R_{d} \approx R_{1}-R_{S}$. Величины сопротивлений $R_{4}, R_{5}$, $R_{6}$ подбираются так, чтобы высокочастотный кабель РК-75 был согласован со стороны моста. Величина сопротивления $R_{7} \gg\left(R_{1}+R_{S}\right)$. Емкость конденсатора $C$ выбирается так, чтобы время $\tau=R_{7} C$ было гораздо больше времени ударно-волнового нагружения сборки. Электронный ключ $K$ заблаговременно (примерно за

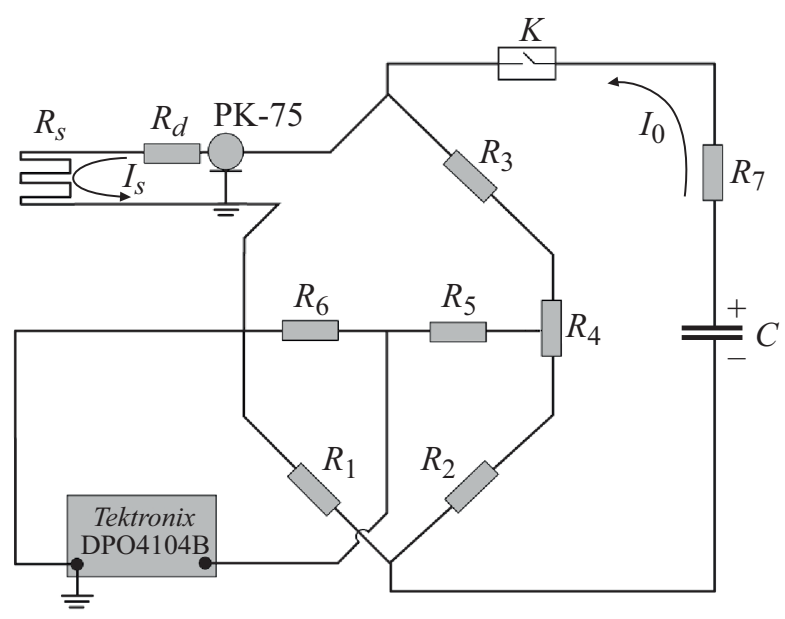

Рис. 3. Электрическая цепь датчика. $R_{S}-$ чувствительный элемент датчика, $I_{S} \approx 3.13 \mathrm{~A}, R_{d}-$ балластное сопротивление, РК-75 - высокочастотный кабель длиной $10 \mathrm{~m}, R_{2}=R_{3}=150 \Omega, R_{4}-$ переменное сопротивление 68, $R_{5}=180, R_{6}=220, R_{7}=91 \Omega, I_{0} \approx 3.16 \mathrm{~A}, C-$ конденсатор емкостью $1000 \mu \mathrm{F}$, предварительно заряженный до $300 \mathrm{~V}$. Входное сопротивление осциллографа Tektronix DPO4104B составляет $1 \mathrm{M} \Omega$. $\sim 20 \mu \mathrm{s})$ замыкается перед моментом нагружения датчика и размыкается спустя $100 \mu \mathrm{s}$.

Переменное сопротивление $R_{4}$ служит для нулевой балансировки моста перед экспериментом. Во время ударно-волнового нагружения происходит разбалансировка моста, которая сопровождается появлением электрического напряжения $U$ на делителе из сопротивлений $R_{5}-R_{6}$, измеряемого высокочастотным осциллографом Tektronix DPO4104B.

Заметим, что поскольку чувствительный элемент датчика вместе с тоководами представляет собой контур с током, то, вообще говоря, разбалансировка моста на рис. 3 происходит не только за счет изменения активного электросопротивления $R_{S}$, но и за счет ЭДС электромагнитной индукции вследствие изменения магнитного потока через контур датчика. В постановке ударноволнового эксперимента по схеме рис. 1 площадь контура и ток $I_{S}$ остаются неизменными во время плоского одномерного ударно-волнового нагружения. Поэтому изменение магнитного потока через контур датчика с током и соответствующее появление ЭДС индукции зависит от степени изменения магнитных свойств чувствительного элемента датчика. Так, например, в случае манганина, состоящего на 85\% из диамагнитной меди, разбалансировка моста происходит только за счет изменения его электросопротивления. Однако в случае железного чувствительного элемента датчика оказывается, что разбалансировка моста происходит не только за счет изменения электросопротивления $R_{S}$, но и под действием ЭДС электромагнитной индукции вследствие изменения магнитного потока при магнитном превращении образца во время ОЦК $\leftrightarrow$ ПУ перехода железа. На рис. 2, $a$ графиком 3 показана типичная осциллограмма $U(t)$ в экспериментах в постановке рис. 1 с чувствительным элементом датчика, изготовленного из железа АРМКО. Обсудим показания датчика на примере этой осциллограммы, а также результаты для реакторной стали 15Х2НМФА.

\section{3. Обсуждение результатов}

Заметим (рис. 2,a), что в момент $t_{1}$ прихода первой ударной волны ступенчатого сжатия давление $P_{1}$ практически равно $P_{1} \approx P_{\alpha}$, где $P_{\alpha}$ - давление начала прямого превращения ОЦК $\rightarrow$ ПУ железа при ударноволновом нагружении из [2]. В этот же момент наблюдается отрицательный отброс $U$ на осциллограмме 3 датчика магнитного превращения. Таким образом, отрицательный импульс $U$ в области $t_{1}$, обусловленный ЭДС электромагнитной индукции при размагничивании образца железа, может служить маркером прямого превращения ОЦК $\rightarrow$ ППУ железа, а давление $P_{1}$ можно рассматривать как давление $P_{\alpha}$ начала этого процесса при ударно-волновом сжатии.

Заметим далее, что при высокочастотных колебаниях 1 после момента $t_{1}$ давление на короткие времена 
Давления начала прямого $P_{\alpha}$ и обратного $P_{\varepsilon}$ превращений, а также их длительности $\tau_{\alpha}$, и $\tau_{\varepsilon}$ в ударно-сжатом поликристаллическом железе АРМКО и корпусной стали 15Х2НМФА

\begin{tabular}{c|c|c|c|c|c|c}
\hline Образец & $P_{\alpha}, \mathrm{GPa}$ & $\tau_{\alpha}, \mathrm{ns}$ & $P_{\varepsilon} \mathrm{GPa}$ & $\tau_{\varepsilon}, \mathrm{ns}$ & Метод & Ссылки \\
\hline ARMКО & $13.9(3)$ & - & $12.3(4)$ & - & манганин & {$[2]$} \\
\hline ARMKO & $12.90-13.74$ & - & $9.8(4)$ & - & VISAR & {$[11]$} \\
\hline ARMKO & 13.9 & 200 & 20.0 & 200 & электромагнитный & настоящая работа \\
\hline 15Кh2NMFA & $>\sim 13 ;<25$ & 300 & 20.0 & 300 & электромагнитный & настоящая работа
\end{tabular}

оказывается меньше давления $P_{\varepsilon}$ обратного превращения ГПУ $\rightarrow$ ОЦК железа и до момента $t_{\alpha \varepsilon}$ колеблется между значениями давления $P_{\alpha}$ и $P_{\varepsilon}$. По-видимому, в этом временном промежутке происходят поочередные $\alpha \rightarrow \varepsilon$ превращения материла датчика между магнитной $\alpha$ - и немагнитной $\varepsilon$-фазами, что обусловливает сложный профиль $U(t)$ в интервале $t_{1}<t<t_{\alpha \varepsilon}$. Однако в момент прихода третьей ступени с амплитудой $P_{2} \approx 20 \mathrm{GPa}$ происходит полное $\alpha \rightarrow \varepsilon$ превращение магнитной фазы $\alpha$ в немагнитную фазу $\varepsilon$, которое сопровождается вторым сильным отрицательным импульсом $U$ длительностью $\Delta t_{\alpha \varepsilon}$. В дальнейшем на интервале $t_{\alpha \varepsilon}<t<t_{\varepsilon \alpha}$ железо находится в состоянии немагнитной фазы $\varepsilon$, площадь контура датчика и ток $I_{S}$ остаются по-прежнему неизменными и соответственно величина сигнала $U$ определяется разбалансировкой моста только за счет изменения активного электросопротивления чувствительного элемента датчика $R_{S}$. Представляется, что время $\Delta t_{\alpha \varepsilon}$ (длительность процесса размагничивания) коррелирует с длительностью $\tau_{\alpha}$ ОЦК $\rightarrow$ ПУ превращения железа и может быть оценено шириной отрицательного импульca $U$, т. е. $\tau_{\alpha} \approx \Delta t_{\alpha \varepsilon}$.

После прохождения через максимум при $40 \mathrm{GPa}$ давление 1 начинает плавно уменьшаться в волне разгрузки. Когда давление достигает величины $P_{3} \approx 20 \mathrm{GPa}$ на осциллограмме 3 в момент $t_{\varepsilon \alpha}$, начинает формироваться положительный импульс $U$, длительность которого составляет $\Delta t_{\varepsilon \alpha}$. Давление $P_{3}$ начала магнитного превращения $\varepsilon \rightarrow \alpha$ разумно соотнести с давлением $P_{\varepsilon}$ начала обратного превращения ГПУ $\rightarrow$ ОЦК при разгрузке железа в ударно-волновом цикле. С этим предположением согласуется тот факт, что в момент $t_{\varepsilon}$ максимума $U$ давление составляет величину $P_{4}$ практически совпадающую с давлением $P_{\varepsilon}=12.3(4) \mathrm{GPa}$ начала обратного превращения ГПУ $\rightarrow$ ОЦК железа в волне разгрузки из[2]. Таким образом, положительный импульс $U$ в области $t_{\varepsilon \alpha}$, обусловленный ЭДС электромагнитной индукции при намагничивании образца железа, может служить маркером обратного превращения ГПУ $\rightarrow$ ОЦК железа, протекающего за время $\Delta t_{\varepsilon \alpha}$. Соответственно время обратного превращения, оцениваемое шириной положительного импульса $U$, составляет величину $\tau_{\varepsilon} \approx \Delta t_{\varepsilon \alpha}$. Отметим, что, как видно на рис. 2, $а$, для железа АРМКО $\tau_{\varepsilon} \approx \tau_{\alpha}=200$ ns. Что же касается различия величин $P_{3}$ и $P_{\varepsilon}$ из [2,11], то можно предположить, что датчик магнитного превращения фиксирует процесс ГПУ $\rightarrow$ ОЦК железа на его более ранних стадиях, чем это делается в предшествующих экспериментах. Однако доказательство этого предположения требует дополнительных исследований.

Итак, сопоставление характеристик ОЦК↔ГПУ перехода железа при ударно-волновом нагружении с показаниями датчика магнитного превращения позволяет предположить, что давления и времена магнитных превращений железа коррелируют с давлениями и временами ОЦК $\leftrightarrow$ ПУ перехода железа при ударно-волновом нагружении. При этом датчик магнитного превращения позволяет установить факт прямого полиморфного ОЦК $\rightarrow$ ГПУ перехода железа, а также давление $P_{\alpha} \approx 13.9 \mathrm{GPa}$ его начала и длительность $\tau_{\alpha} \approx 200 \mathrm{~ns}$ этого процесса. Датчик позволяет также для железа установить факт обратного ГПУ $\rightarrow$ ОЦК перехода и его длительность $\tau_{\varepsilon} \approx \tau_{\alpha}$.

В логике полученных результатов для железа обсудим теперь эксперименты для реакторной стали (рис. 2,b). На рис. 2,b графиком 1 показан расчетный профиль давления в чувствительном элементе датчика, изготовленном из реакторной стали 15Х2НМФА. Поскольку массовое содержание легирующих добавок в стали невелико, составляя не более 5\%, то, как и выше, расчет был проведен с уравнением состояния для железа, но с использованием упруго-пластических характеристик реакторной стали из[13]. Постановка эксперимента со сталью 15Х2НМФА идентична таковой для железа АРМКО. Типичная экспериментальная осциллограмма представлена графиком 3 на рис. 2,b. Как видно, эта осциллограмма содержит отрицательный и положительный импульсы $U$. Это означает, что аналогично железу сталь 15Х2НМФА испытывает обратимый полиморфный ОЦК $\leftrightarrow$ ПУ переход при ударно-волновом нагружении. Однако некоторые параметры этого перехода для стали отличаются от таковых для железа. Во-первых, первая ударная волна амплитудой $P_{1} \approx 13 \mathrm{GPa}$ не сопровождается появлением отрицательного импульса $U$. Отрицательный импульс $U$ формируется лишь при давлении $P_{2} \approx 25 \mathrm{GPa}$. Это означает, что давление $P_{\alpha}$ начала пря-

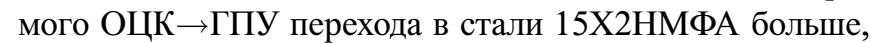
чем в железе и может быть охарактеризовано соотно- 
шением $\sim 13<P_{\alpha}<25 \mathrm{GPa}$. Во-вторых, длительность прямого перехода в стали составляет $\tau_{\varepsilon} \approx 300 \mathrm{~ns}$, что в полтора раза превышает аналогичную характеристику для железа. Остальные характеристики - давление $P_{3}$ начала обратного ГПУ $\rightarrow$ ОЦК перехода $P_{\varepsilon}$ и соотношение между длительностью прямого $\tau_{\alpha}$ и обратного $\tau_{\varepsilon}$ переходов в стали совпадают с таковыми для железа.

Давления начала прямого и обратного перехода, а также их длительности для железа и стали 15Х2НМФА при ударном сжатии в сравнении с литературными данными сведены в таблице.

\section{Заключение}

Разработан датчик магнитного превращения для исследования полиморфного перехода в ферромагнитных сталях. Методика основана на использовании сопутствующего электромагнитного импульса в качестве индика-

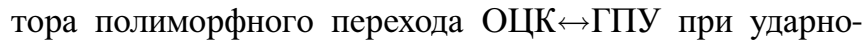
волновом нагружении сталей. Зарегистрированы магнитные превращения во время прямого и обратного полиморфного перехода при плоском одномерном ударноволновом нагружении железа АРМКО. Показано, что отрицательный и положительный сигналы датчика коррелируют соответственно с прямым ОЦК↔ГПУ и обратным ГПУ $\leftrightarrow$ ОЦ полиморфными переходами железа. На основании показаний датчика магнитного превращения для корпусной стали 15Х2НМФА идентифицирован ее обратимый полиморфный ОЦК↔ГПУ переход, давление и длительность которого превышает аналогичные характеристики для железа АРМКО.

\section{Финансирование работы}

Работа выполнена при поддержке Государственной корпорации „Росатом“ с использованием оборудования Уникальной научной установки „Экспериментальный взрывной стенд“ по теме Государственного задания № AAAA-A19-119071190040-5.

\section{Конфликт интересов}

Авторы заявляют, что у них нет конфликта интересов.

\section{Список литературы}

[1] D. Bancroft, E.L. Peterson, S. Minshall. J. Appl. Phys., 27, 291 (1956).

[2] А.В. Ананьин, А.Н. Дрёмин, Г.И. Канель. ФГВ, 17 (3), 437 (1981).

[3] X. Liu, T. Mashimo, N. Kawai, T. Sano, X. Zhou. J. Appl. Phys., 124, 215101 (2018).

[4] J. Hawreliak, J.D. Colvin, J.H. Eggert, D.H. Kalantar, H.E. Lorenzana, J.S. Stölken, H.M. Davies, T.C. Germann, B.L. Holian, K. Kadau, P.S. Lomdahl, A. Higginbotham, K. Rosolankova, J. Sheppard, J.S. Wark. Phys. Rev. B, 74 184107 (2006).
[5] R.N. Keeler, A.C. Mitchell. Sol. St. Com., 7, 271 (1969)

[6] Y. Bi, H. Tan, F. Jing. J. Phys.: Condens. Matter., 14, 10849 (2002).

[7] С.С. Набатов, А.Н. Дрёмин, В.И. Постнов, В.В. Якушев. ПЖЭТФ, 29 (7), 407 (1979).

[8] А.М. Молодец, А.А. Голышев. ФТТ, 61 (8), 1492 (2019).

[9] А.М. Молодец. ФТТ, 55 (11), 2090 (2013).

[10] М.Л. Уилкинс. Вычислительные методы в гидродинамике (Мир, М., 1967), с. 212. [Пер. с англ.: M.L. Wilkins, in: Methods of Computational Physics, ed. by B. Alder, S. Fernbach, M. Rotenberg. (Academic, NY., 1964), vol. 3, p. 211.]

[11] L.M. Barker, R.E. Hollenbach. J. Appl. Phys., 45 (11), 4872 (1974).

[12] Los Alamos Shock wave Profile data, Editor Charles E. Morris, University of California Press, Berkeley, Los Angeles, London, 1982, ISBN 0-520-04007-4.

[13] Г.И. Канель, Г.В. Гаркушин, А.С. Савиных, С.В. Разоренов, С.А. Атрошенко. ЖТФ, 90 (3), 441 (2020). 